Supporting Information

\title{
Continuous Synthesis of Methanol from Methane and Steam over Copper-mordenite
}

Yu Ri Jeong ${ }^{\dagger}$, Hyeonjung Jung $\$$, Jongkyu Kang ${ }^{\dagger}$, Jeong Woo Han ${ }^{\ddagger *}$, and Eun Duck Park ${ }^{\dagger *}$

'Department of Chemical Engineering and Department of Energy Systems Research, Ajou University 206 World cup-ro, Yeongtong-Gu, Suwon 16499, Republic of Korea.

*Department of Chemical Engineering, Pohang University of Science and Technology (POSTECH), 77

Chengam-ro, Nam-Gu, Pohang 37673, Republic of Korea.

*Corresponding author: Jeong Woo Han: jwhan@postech.ac.kr; Eun Duck Park: edpark@ajou.ac.kr 


\section{Materials and Methods}

\section{$\underline{\text { S1.1. Commercial zeolites }}$}

Various H-mordenites such as H-mordenite (7.5) (HSZ-620HOA, $\mathrm{SiO}_{2} / \mathrm{Al}_{2} \mathrm{O}_{3}=15$ ), H-mordenite (9) (HSZ640HOA, $\mathrm{SiO}_{2} / \mathrm{Al}_{2} \mathrm{O}_{3}=18$ ), H-mordenite (15) (HSZ-660HOA, $\mathrm{SiO}_{2} / \mathrm{Al}_{2} \mathrm{O}_{3}=30$ ), and Na-mordenite (9) (HSZ642NAA, $\mathrm{SiO}_{2} / \mathrm{Al}_{2} \mathrm{O}_{3}=18$ ) were purchased from Tosoh.

\section{S1.2. Dealumination of H-mordenite (15)}

The parent material was an H-mordenite (15). Nitric acid was used as a dealuminating agent. The acid-treatment of the parent mordenite was carried out under two different conditions. H-mordenite (25) and H-mordenite (35) were prepared using $2 \mathrm{M}$ nitric acid at $90{ }^{\circ} \mathrm{C}$ for $4 \mathrm{~h}$ and $6 \mathrm{M}$ nitric acid at $90{ }^{\circ} \mathrm{C}$ for $8 \mathrm{~h}$, respectively. The volume $(\mathrm{V}$ in $\mathrm{mL}$ ) of the nitric acid solution used was equal to 10 times the weight ( $\mathrm{P}$ in $\mathrm{g})$ of $\mathrm{H}$-mordenite $(\mathrm{V} / \mathrm{P}=10)$. After an acid-treatment, the recovered zeolite was washed with deionized water several times, dried overnight at $110^{\circ} \mathrm{C}$, and then calcined in air at $400{ }^{\circ} \mathrm{C}$ for $3 \mathrm{~h}$.

\section{S1.3. Cu ion-exchange}

The $\mathrm{Cu}$-mordenite was prepared with an aqueous ion-exchange of $\mathrm{H}$-mordenite (or Na-mordenite) and $\mathrm{Cu}$ (II) acetate monohydrate $\left(\mathrm{Cu}\left(\mathrm{CH}_{3} \mathrm{COO}\right)_{2} \cdot \mathrm{H}_{2} \mathrm{O}\right.$, Kanto Chemical Co.) at room temperature. H-mordenite was stirred in a aqueous solution of $\mathrm{Cu}$ acetate whose concentration was controlled in the range of $0.1 \sim 1 \mathrm{mM}$. After $24 \mathrm{~h}$, the resultant slurry was filtered and the cake was dried at $110^{\circ} \mathrm{C}$ for $12 \mathrm{~h}$. To obtain a higher $\mathrm{Cu}$ content, the ion exchange/filteration procedure was repeated twice or three times. The final powder was calcined in air at $400{ }^{\circ} \mathrm{C}$ for $3 \mathrm{~h}$.

\section{S1.4. Characterization of catalyst}

Elemental analysis. Inductively-coupled plasma-optical emission spectroscopy (ICP-OES) was performed using a OPTIMA 5300DV instrument to determine the metal content of the sample such as sodium (Na), aluminum (Al), copper $(\mathrm{Cu})$. Also, Silicon $(\mathrm{Si})$ contents was determined using wavelength dispersive X-ray fluorescence spectrometer (WD-XRF) (Rigaku ZSX Primus).

Surface area and pore volume quantification. The BET surface areas of the catalysts were calculated by Brunauer-Emmett-Teller (BET) and External surface area and micropore volume were determined by t-plot method using $\mathrm{N}_{2}$ adsorption-desorption isotherms measured on a Micromeritics ASAP2020 system. Prior to the measurement, the samples were degassed under vacuum at $200{ }^{\circ} \mathrm{C}$ for $4 \mathrm{~h}$.

Temperature programmed surface reaction with mass spectroscopy (TPSR-MS). TPSR-MS was performed on Micromeritics Autochem 2920 instrument equipped with a mass spectrometer (Cirrus ${ }^{\mathrm{TM}} 2$, MKS). The sample was pretreated with air at $400{ }^{\circ} \mathrm{C}$ for $1 \mathrm{~h}$ and then heated from 30 to $100{ }^{\circ} \mathrm{C}$ with a heating rate of $10{ }^{\circ} \mathrm{C} \mathrm{min}-1$ by flowing methane at a flowrate of $30 \mathrm{~mL} / \mathrm{min}$. The effluent stream was analyzed while cofeeding $\mathrm{CH}_{4}$ and water vapor with increasing the reaction temperature from 100 to $400{ }^{\circ} \mathrm{C}$ at a heating rate of $10{ }^{\circ} \mathrm{C} \mathrm{min}^{-1}$. After the temperature reached $400{ }^{\circ} \mathrm{C}$, the product stream was further analyzed for $1 \mathrm{~h}$ while maintaining the temperature at $400{ }^{\circ} \mathrm{C}$. During the analysis, a gaseous methane went at a flowrate of $30 \mathrm{~mL} / \mathrm{min}$ through the evaporator filled with distilled water at $73{ }^{\circ} \mathrm{C}$. The ion signals recorded at $\mathrm{m} / \mathrm{z}=31$ and 44 were used for monitoring methanol and carbon dioxide, respectively.

\section{S1.5. Catalytic activity}

The continuous methanol synthesis was carried out in a packed-bed reactor. The catalyst was loaded in a fixedbed stainless-steel reactor (O.D. $=9.525 \mathrm{~mm}$, I.D. $=7.745 \mathrm{~mm}$ ) and was supported by quartz wool. The flowrate of each gas was controlled by a mass flow controller (Brooks instruments). The reaction temperature was monitored with a thermocouple located just above the catalyst bed. The steam was generated in a vaporizer maintained at $200^{\circ} \mathrm{C}$. The flowrate of the liquid water fed into the vaporizer was controlled with a syringe pump (ISCO MODEL 260D). Typically, $0.80 \mathrm{~g}$ of catalyst was contacted with the feed gas composed of $33 \mathrm{~mol} \%$ methane and $67 \%$ steam at a total flow rate of $240 \mathrm{~mL} / \mathrm{min}$ at standard temperature and pressure. The liquid product was obtained with a condenser maintained at $1{ }^{\circ} \mathrm{C}$ and analyzed by ${ }^{1} \mathrm{H}-\mathrm{NMR}$ spectroscopy, recorded at room temperature on a Jeol Resonance ECZ600R $(1 \mathrm{H} 599.7 \mathrm{MHz})$, and quantified with a $0.1 \%$ trimethylsilylpropanoic acid (TMSP)/ $\mathrm{D}_{2} \mathrm{O}$ standard (Euriso-top). Typically, $0.7 \mathrm{~mL}$ of sample and $0.1 \mathrm{~mL}$ of $\mathrm{TMSP} / \mathrm{D}_{2} \mathrm{O}$ were placed in an NMR tube along with the standard. A solvent suppression program was run to minimize the signal arising from the solvent. The gas product was analyzed by a gas chromatography (GC, YL 
6500) equipped with a thermal conductivity detector (TCD), methanizer, and flame ionization detector (FID). The products were separated using packed column (Carbosphere) and quantified with a TCD and FID.

\section{S1.6. Computational detail}

All DFT-D3 calculations were carried out by Vienna ab Initio Simulation Package. The projector augmented wave pseudopotentials method was used to describe electron-ion interactions. The Perdew-Burke-Ernzerhof exchange correlation functional with spin polarization was used. The Brillouin zone integrations were performed using a 1 $\times 1 \times 1$ Monkhorst-Pack grid. During the structure optimization, all energies change criterion was set to $10^{-4} \mathrm{eV}$, the atoms were relaxed until the force acting on each atom was less than $0.03 \mathrm{eV} \AA^{-1}$, and the plane wave cutoff was set to $400 \mathrm{eV}$. 


\section{Results and Discussion}

Table S1. The physicochemical properties of $\mathrm{Cu}-\mathrm{MOR}$ catalysts.

\begin{tabular}{|c|c|c|c|c|c|c|}
\hline Entry & Catalyst $^{\mathrm{a}}$ & $\begin{array}{l}\mathrm{Si} / \mathbf{A l} \\
\text { ratio }^{\mathrm{b}}\end{array}$ & $\begin{array}{l}\mathrm{Cu} / \mathrm{Al} \\
\operatorname{ratio}^{\mathrm{c}}\end{array}$ & $\begin{array}{c}\text { BET surface } \\
\text { area }^{\mathrm{d}} \\
\left(\mathrm{m}^{2} / \mathrm{g}\right)\end{array}$ & $\begin{array}{c}\text { External surface } \\
\operatorname{area}^{\mathrm{e}} \\
\left(\mathrm{m}^{2} / \mathrm{g}\right)\end{array}$ & $\begin{array}{c}\text { Micropore } \\
\text { volume }^{\mathrm{e}} \\
\left(\mathrm{cm}^{3} / \mathrm{g}\right)\end{array}$ \\
\hline 1 & $3.3 \% \mathrm{Cu}-\mathrm{Na}-\mathrm{MOR}(9)$ & 9 & 0.389 & 411 & 33.1 & 0.17 \\
\hline 2 & $3.3 \%$ Cu-H-MOR (9) & 9 & 0.422 & 485 & 39.0 & 0.21 \\
\hline 3 & $2.5 \% \mathrm{Cu}-\mathrm{H}-\mathrm{MOR}(7.5)$ & 7.5 & 0.373 & 445 & 31.8 & 0.19 \\
\hline 4 & $1.8 \% \mathrm{Cu}-\mathrm{H}-\mathrm{MOR}(7.5)$ & 7.5 & 0.176 & 393 & 19.4 & 0.17 \\
\hline 5 & $1.5 \% \mathrm{Cu}-\mathrm{H}-\mathrm{MOR}(7.5)$ & 7.5 & 0.145 & 439 & 24.8 & 0.19 \\
\hline 6 & $1.1 \% \mathrm{Cu}-\mathrm{H}-\mathrm{MOR}(7.5)$ & 7.5 & 0.109 & 448 & 25.3 & 0.20 \\
\hline 7 & $0.5 \% \mathrm{Cu}-\mathrm{H}-\mathrm{MOR}(7.5)$ & 7.5 & 0.051 & 441 & 29.9 & 0.19 \\
\hline 8 & $2.7 \% \mathrm{Cu}-\mathrm{H}-\mathrm{MOR}(15)$ & 15 & 0.540 & 447 & 35.9 & 0.19 \\
\hline 9 & $1.6 \% \mathrm{Cu}-\mathrm{H}-\mathrm{MOR}(15)$ & 15 & 0.263 & 498 & 42.3 & 0.21 \\
\hline 10 & $1.1 \% \mathrm{Cu}-\mathrm{H}-\mathrm{MOR}(15)$ & 15 & 0.185 & 423 & 34.4 & 0.18 \\
\hline 11 & $0.6 \% \mathrm{Cu}-\mathrm{H}-\mathrm{MOR}(15)$ & 15 & 0.095 & 489 & 23.4 & 0.22 \\
\hline 12 & $0.3 \% \mathrm{Cu}-\mathrm{H}-\mathrm{MOR}(15)$ & 15 & 0.053 & 446 & 35.4 & 0.19 \\
\hline 13 & $0.4 \% \mathrm{Cu}-\mathrm{H}-\mathrm{MOR}(25)$ & 25 & 0.109 & 453 & 47.4 & 0.19 \\
\hline 14 & $0.2 \% \mathrm{Cu}-\mathrm{H}-\mathrm{MOR}(35)$ & 35 & 0.103 & 501 & 46.8 & 0.21 \\
\hline
\end{tabular}

${ }^{a}$ Catalyst is denoted as $\mathrm{x} \% \mathrm{Cu}-\mathrm{MOR}(\mathrm{y})$, $\mathrm{x}$ means $\mathrm{Cu}$ content $(\mathrm{wt} \%)$ and y means $\mathrm{Si} / \mathrm{Al}$ ratio of parent zeolite. ${ }^{\mathrm{b}} \mathrm{Si} / \mathrm{Al}$ ratio is the value supplied by the zeolite suppliers.

${ }^{\mathrm{c}} \mathrm{Cu} / \mathrm{Al}$ ratio and metal content were determined by ICP-OES.

${ }^{\mathrm{d} B E T}$ surface area was measured by $\mathrm{N}_{2}$ physisorption.

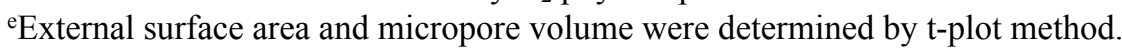


Table S2. Catalytic activities for anaerobic methane oxidation with steam over $\mathrm{Cu}-\mathrm{H}-\mathrm{MOR}$ and $\mathrm{Cu}-\mathrm{Na}-\mathrm{MOR}$. $^{\mathrm{a}}$

\begin{tabular}{cccccc}
\hline Entry & Catalyst & $\begin{array}{c}\text { Temp. } \\
\left({ }^{\circ} \mathbf{C}\right)\end{array}$ & $\begin{array}{c}\mathbf{C H}_{3} \mathbf{O H} \\
\text { Formation rate } \\
(\mathbf{m m o l} / \mathbf{m o l ~ C u} / \mathbf{h})\end{array}$ & $\begin{array}{c}\mathbf{C O}_{\mathbf{2}} \\
\text { Formation rate } \\
(\mathbf{m m o l} / \mathbf{m o l ~ C u} / \mathbf{h})\end{array}$ & $\begin{array}{c}\mathbf{C H}_{\mathbf{3}} \mathbf{O H} \\
\text { Selectivity } \\
(\mathbf{\%})\end{array}$ \\
\hline 1 & $3.3 \% \mathrm{Cu}-\mathrm{H}-\mathrm{MOR}(9)$ & 350 & $7.22 \pm 1.8$ & 0 & 100 \\
2 & $3.3 \% \mathrm{Cu}-\mathrm{H}-\mathrm{MOR}(9)$ & 400 & $6.28 \pm 0.8$ & 0 & 100 \\
3 & $3.3 \% \mathrm{Cu}-\mathrm{Na}-\mathrm{MOR}(9)$ & 350 & $5.77 \pm 0.9$ & $0.69 \pm 0.3$ & $89.7 \pm 2.3$ \\
4 & $3.3 \% \mathrm{Cu}-\mathrm{Na}-\mathrm{MOR}(9)$ & 400 & $2.71 \pm 1.1$ & $2.14 \pm 0.4$ & $54.0 \pm 6.1$ \\
\hline
\end{tabular}

aFeed composition: $33 \mathrm{~mol} \%$ methane and $67 \mathrm{~mol} \%$ steam. 
Table S3. Catalytic activities for methane oxidation with steam over $\mathrm{Cu}-\mathrm{H}-\mathrm{MOR}$ and $\mathrm{Cu}-\mathrm{Na}-\mathrm{MOR}$. ${ }^{\mathrm{a}}$

\begin{tabular}{cccccc}
\hline Entry & Catalyst & $\begin{array}{c}\text { Temp. } \\
\left({ }^{\circ} \mathbf{C}\right)\end{array}$ & $\begin{array}{c}\mathbf{C H}_{\mathbf{3}} \mathbf{O H} \\
\text { Formation rate } \\
(\mathbf{m m o l} / \mathbf{m o l ~ C u} / \mathbf{h})\end{array}$ & $\begin{array}{c}\mathbf{C O}_{2} \\
\text { Formation rate } \\
(\mathbf{m m o l} / \mathbf{m o l ~ C u} / \mathbf{h})\end{array}$ & $\begin{array}{c}\mathbf{C H}_{\mathbf{3}} \mathbf{O H} \\
\text { Selectivity } \\
(\mathbf{\%})\end{array}$ \\
\hline 1 & $3.3 \% \mathrm{Cu}-\mathrm{H}-\mathrm{MOR}(9)$ & 350 & $29.3 \pm 4.8$ & $5.3 \pm 0.9$ & 84.7 \\
2 & $3.3 \% \mathrm{Cu}-\mathrm{H}-\mathrm{MOR}(9)$ & 400 & $22.6 \pm 3.1$ & $11.3 \pm 3.5$ & $66.7 \pm 4.0$ \\
3 & $3.3 \% \mathrm{Cu}-\mathrm{Na}-\mathrm{MOR}(9)$ & 350 & $22.9 \pm 4.0$ & $12.5 \pm 2.2$ & $64.7 \pm 0.1$ \\
4 & $3.3 \% \mathrm{Cu}-\mathrm{Na}-\mathrm{MOR}(9)$ & 400 & $11.4 \pm 1.2$ & $17.9 \pm 5.3$ & $38.9 \pm 4.7$ \\
\hline
\end{tabular}

aFeed composition: $33 \mathrm{~mol} \%$ methane, $85 \mathrm{ppm} \mathrm{O}_{2}$, and $67 \mathrm{~mol} \%$ steam. 
Table S4. Catalytic activities for anaerobic methane oxidation with steam over $\mathrm{Cu}-\mathrm{H}-\mathrm{MOR}(7.5)$ and $\mathrm{Cu}-\mathrm{Na}-$ $\operatorname{MOR}(15) .^{\mathrm{a}}$

\begin{tabular}{|c|c|c|c|c|c|}
\hline Entry & Catalyst & $\begin{array}{c}\text { Temp. } \\
\left({ }^{\circ} \mathrm{C}\right)\end{array}$ & $\begin{array}{c}\mathrm{CH}_{3} \mathrm{OH} \\
\text { Formation rate } \\
(\mathrm{mmol} / \mathrm{mol} \mathrm{Cu} / \mathrm{h})\end{array}$ & $\begin{array}{c}\mathrm{CO}_{2} \\
\text { Formation rate } \\
(\mathrm{mmol} / \mathrm{mol} \mathrm{Cu} / \mathrm{h})\end{array}$ & $\begin{array}{c}\mathrm{CH}_{3} \mathrm{OH} \\
\text { Selectivity } \\
(\%)\end{array}$ \\
\hline 1 & $2.5 \% \mathrm{Cu}-\mathrm{H}-\mathrm{MOR}(7.5)$ & 350 & $10.7 \pm 1.7$ & 0 & 100 \\
\hline 2 & $2.5 \% \mathrm{Cu}-\mathrm{H}-\mathrm{MOR}(7.5)$ & 400 & $12.6 \pm 0.4$ & 0 & 100 \\
\hline 3 & $1.8 \% \mathrm{Cu}-\mathrm{H}-\mathrm{MOR}$ (7.5) & 350 & $9.83 \pm 1.31$ & 0 & 100 \\
\hline 4 & $1.8 \% \mathrm{Cu}-\mathrm{H}-\mathrm{MOR}$ (7.5) & 400 & $13.96 \pm 1.78$ & 0 & 100 \\
\hline 5 & $1.5 \% \mathrm{Cu}-\mathrm{H}-\mathrm{MOR}$ (7.5) & 350 & $16.8 \pm 2.4$ & 0 & 100 \\
\hline 6 & $1.5 \% \mathrm{Cu}-\mathrm{H}-\mathrm{MOR}(7.5)$ & 400 & $18.0 \pm 2.9$ & 0 & 100 \\
\hline 7 & $1.1 \% \mathrm{Cu}-\mathrm{H}-\mathrm{MOR}$ (7.5) & 350 & $16.0 \pm 7.0$ & 0 & 100 \\
\hline 8 & $1.1 \% \mathrm{Cu}-\mathrm{H}-\mathrm{MOR}$ (7.5) & 400 & $17.0 \pm 2.6$ & 0 & 100 \\
\hline 9 & $0.5 \% \mathrm{Cu}-\mathrm{H}-\mathrm{MOR}(7.5)$ & 350 & Not detected & 0 & 100 \\
\hline 10 & $0.5 \% \mathrm{Cu}-\mathrm{H}-\mathrm{MOR}(7.5)$ & 400 & $10.9 \pm 6.1$ & 0 & 100 \\
\hline 11 & $2.7 \% \mathrm{Cu}-\mathrm{H}-\mathrm{MOR}(15)$ & 350 & $5.9 \pm 0.2$ & 0 & 100 \\
\hline 12 & $2.7 \% \mathrm{Cu}-\mathrm{H}-\mathrm{MOR}(15)$ & 400 & $8.8 \pm 1.5$ & 0 & 100 \\
\hline 13 & $1.6 \% \mathrm{Cu}-\mathrm{H}-\mathrm{MOR}(15)$ & 350 & $13.9 \pm 2.0$ & 0 & 100 \\
\hline 14 & $1.6 \% \mathrm{Cu}-\mathrm{H}-\mathrm{MOR}(15)$ & 400 & $14.2 \pm 1.3$ & 0 & 100 \\
\hline 15 & $1.1 \% \mathrm{Cu}-\mathrm{H}-\mathrm{MOR}(15)$ & 350 & $20.8 \pm 2.6$ & 0 & 100 \\
\hline 16 & $1.1 \% \mathrm{Cu}-\mathrm{H}-\mathrm{MOR}(15)$ & 400 & $17.5 \pm 1.9$ & 0 & 100 \\
\hline 17 & $0.6 \% \mathrm{Cu}-\mathrm{H}-\mathrm{MOR}(15)$ & 350 & $10.3 \pm 1.5$ & 0 & 100 \\
\hline 18 & $0.6 \% \mathrm{Cu}-\mathrm{H}-\mathrm{MOR}(15)$ & 400 & $8.9 \pm 0.6$ & 0 & 100 \\
\hline 19 & $0.3 \% \mathrm{Cu}-\mathrm{H}-\mathrm{MOR}(15)$ & 350 & Not detected & 0 & 100 \\
\hline 20 & $0.3 \% \mathrm{Cu}-\mathrm{H}-\mathrm{MOR}(15)$ & 400 & Not detected & 0 & 100 \\
\hline
\end{tabular}

aFeed composition: $33 \mathrm{~mol} \%$ methane and $67 \mathrm{~mol} \%$ steam. 
Table S5. Catalytic activities for methane oxidation with steam over Cu-H-MOR (7.5) and Cu-Na-MOR (15). ${ }^{\mathrm{a}}$

\begin{tabular}{|c|c|c|c|c|c|}
\hline Entry & Catalyst & $\begin{array}{c}\text { Temp. } \\
\left({ }^{\circ} \mathrm{C}\right)\end{array}$ & $\begin{array}{c}\mathrm{CH}_{3} \mathrm{OH} \\
\text { Formation rate } \\
(\mathrm{mmol} / \mathrm{mol} \mathrm{Cu} / \mathrm{h})\end{array}$ & $\begin{array}{c}\mathrm{CO}_{2} \\
\text { Formation rate } \\
(\mathrm{mmol} / \mathrm{mol} \mathrm{Cu} / \mathrm{h})\end{array}$ & $\begin{array}{c}\mathrm{CH}_{3} \mathrm{OH} \\
\text { Selectivity } \\
(\%)\end{array}$ \\
\hline 1 & $2.5 \% \mathrm{Cu}-\mathrm{H}-\mathrm{MOR}(7.5)$ & 350 & $58.6 \pm 4.8$ & $3.3 \pm 1.2$ & $94.7 \pm 1.5$ \\
\hline 2 & $2.5 \% \mathrm{Cu}-\mathrm{H}-\mathrm{MOR}(7.5)$ & 400 & $40.4 \pm 2.5$ & $5.2 \pm 1.1$ & $88.7 \pm 1.5$ \\
\hline 3 & $1.5 \% \mathrm{Cu}-\mathrm{H}-\mathrm{MOR}(7.5)$ & 350 & $57.5 \pm 7.3$ & $0.9 \pm 0.5$ & $98.5 \pm 0.7$ \\
\hline 4 & $1.5 \% \mathrm{Cu}-\mathrm{H}-\mathrm{MOR}(7.5)$ & 400 & $85.3 \pm 0.6$ & $2.7 \pm 2.2$ & $97.0 \pm 2.4$ \\
\hline 5 & $1.1 \% \mathrm{Cu}-\mathrm{H}-\mathrm{MOR}(7.5)$ & 350 & $88.9 \pm 20.1$ & $0.7 \pm 1.1$ & $99.2 \pm 1.1$ \\
\hline 6 & $1.1 \% \mathrm{Cu}-\mathrm{H}-\mathrm{MOR}(7.5)$ & 400 & $98.2 \pm 5.9$ & $0.9 \pm 0.5$ & $99.1 \pm 0.5$ \\
\hline 7 & $0.5 \% \mathrm{Cu}-\mathrm{H}-\mathrm{MOR}(7.5)$ & 350 & $48.7 \pm 14.1$ & 0 & 100 \\
\hline 8 & $0.5 \% \mathrm{Cu}-\mathrm{H}-\mathrm{MOR}(7.5)$ & 400 & $27.5 \pm 24.5$ & 0 & 100 \\
\hline 9 & $2.7 \% \mathrm{Cu}-\mathrm{H}-\mathrm{MOR}(15)$ & 350 & $38.0 \pm 3.1$ & $10.0 \pm 0.5$ & $79.2 \pm 0.6$ \\
\hline 10 & $2.7 \% \mathrm{Cu}-\mathrm{H}-\mathrm{MOR}(15)$ & 400 & $12.8 \pm 1.2$ & $24.9 \pm 3.8$ & $34.0 \pm 1.4$ \\
\hline 11 & $1.6 \% \mathrm{Cu}-\mathrm{H}-\mathrm{MOR}(15)$ & 350 & $92.7 \pm 6.5$ & $1.1 \pm 0.5$ & $98.8 \pm 0.4$ \\
\hline 12 & $1.6 \% \mathrm{Cu}-\mathrm{H}-\mathrm{MOR}(15)$ & 400 & $77.2 \pm 1.9$ & $3.3 \pm 1.9$ & $95.9 \pm 2.1$ \\
\hline 13 & $1.1 \% \mathrm{Cu}-\mathrm{H}-\mathrm{MOR}(15)$ & 350 & $125 \pm 3.1$ & $0.4 \pm 0.1$ & $99.7 \pm 0.1$ \\
\hline 14 & $1.1 \% \mathrm{Cu}-\mathrm{H}-\mathrm{MOR}(15)$ & 400 & $143 \pm 1.9$ & $0.7 \pm 0.6$ & $99.5 \pm 0.4$ \\
\hline 15 & $0.6 \% \mathrm{Cu}-\mathrm{H}-\mathrm{MOR}(15)$ & 350 & $100 \pm 8.2$ & 0 & 100 \\
\hline 16 & $0.6 \% \mathrm{Cu}-\mathrm{H}-\mathrm{MOR}(15)$ & 400 & $125 \pm 17.3$ & 0 & 100 \\
\hline 17 & $0.3 \% \mathrm{Cu}-\mathrm{H}-\mathrm{MOR}(15)$ & 350 & $62.9 \pm 10.8$ & 0 & 100 \\
\hline 18 & $0.3 \% \mathrm{Cu}-\mathrm{H}-\mathrm{MOR}(15)$ & 400 & $46.6 \pm 15.6$ & 0 & 100 \\
\hline
\end{tabular}

aFeed composition: $33 \mathrm{~mol} \%$ methane, $85 \mathrm{ppm} \mathrm{O}_{2}$, and $67 \mathrm{~mol} \%$ steam. 

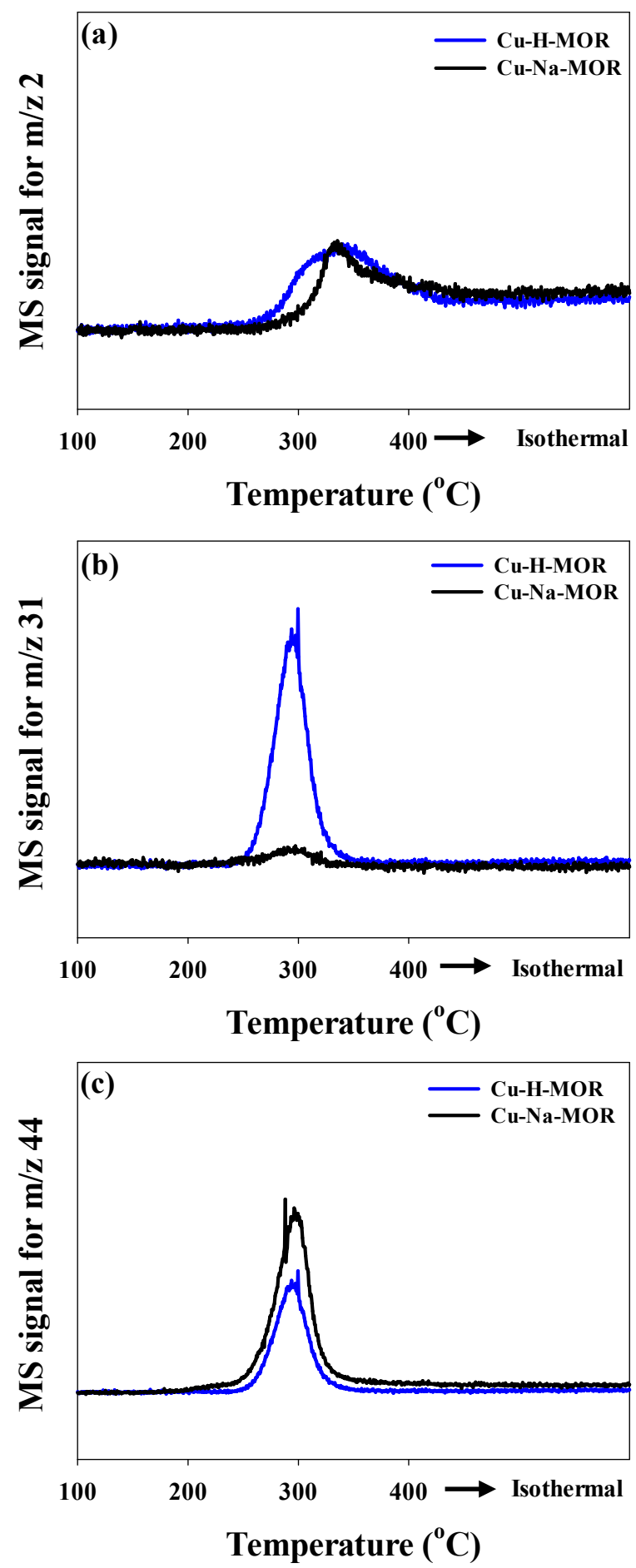

Figure S1. Temperature-programmed surface reactions with mass spectroscopy (TPSRs-MS) patterns over $3.3 \% \mathrm{Cu}-\mathrm{H}-\mathrm{MOR}$ (9) and 3.3\% Cu-Na-MOR (9). (a) $\mathrm{m} / \mathrm{z}=2$ (hydrogen), (b) $\mathrm{m} / \mathrm{z}=31$ (methanol), (c) $\mathrm{m} / \mathrm{z}=44$ (carbon dioxide). 


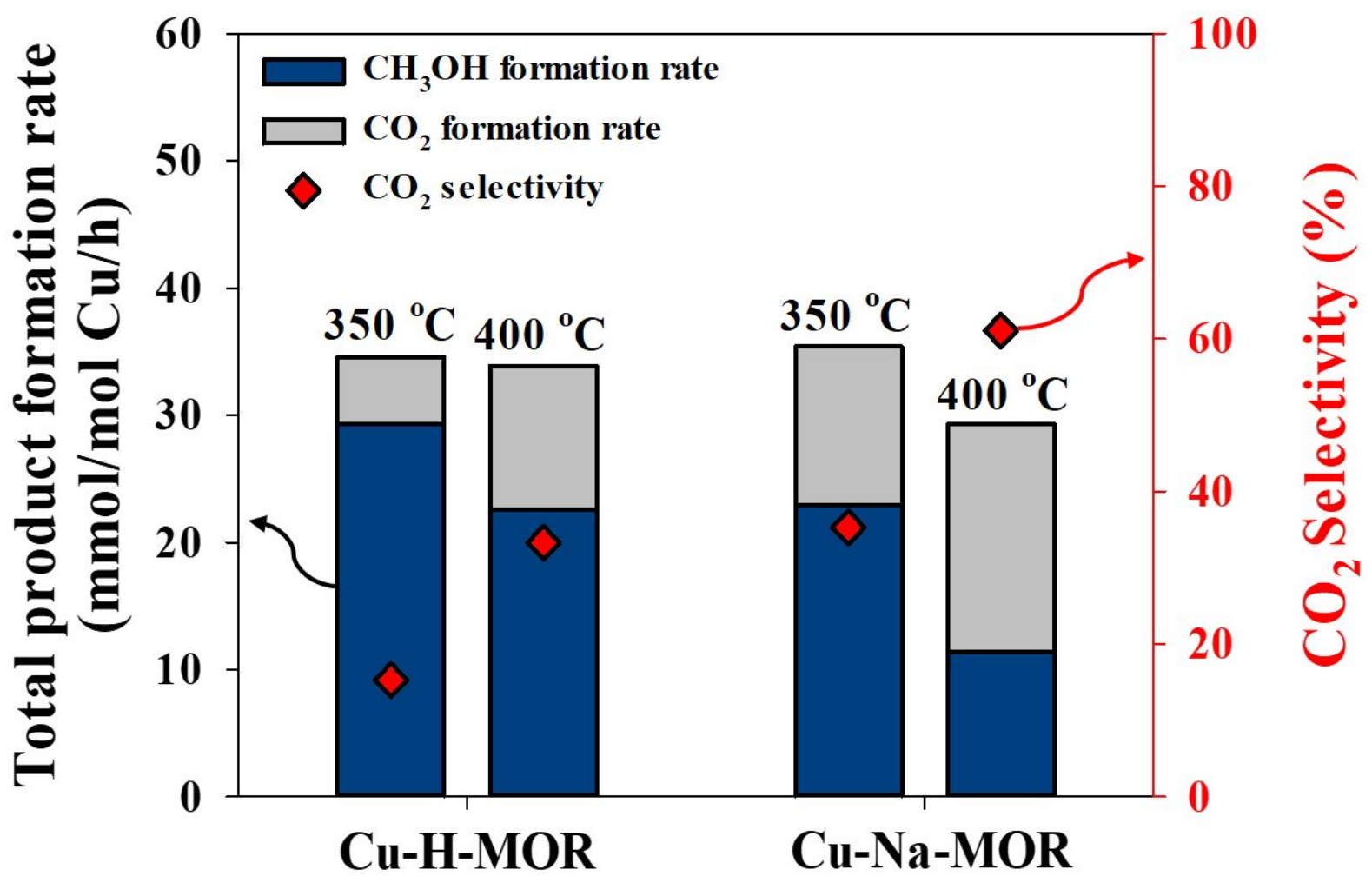

Figure S2. Continuous methane conversion into methanol over 3.3\% Cu-H-MOR(9) and 3.3\% Cu-Na-MOR(9) with steam and dioxygen. Bar chart shows total product production rate. Blue and gray represent formation rates of methanol and carbon dioxide, respectively. Red dots indicate carbon dioxide selectivity. Reaction temperatures were 350 and $400{ }^{\circ} \mathrm{C}$. Feed was composed of $33 \mathrm{~mol} \%$ methane, $85 \mathrm{ppm} \mathrm{O}$, and $67 \mathrm{~mol} \%$ steam. 
(a) $\mathrm{Si} / \mathrm{Al}=7.5$
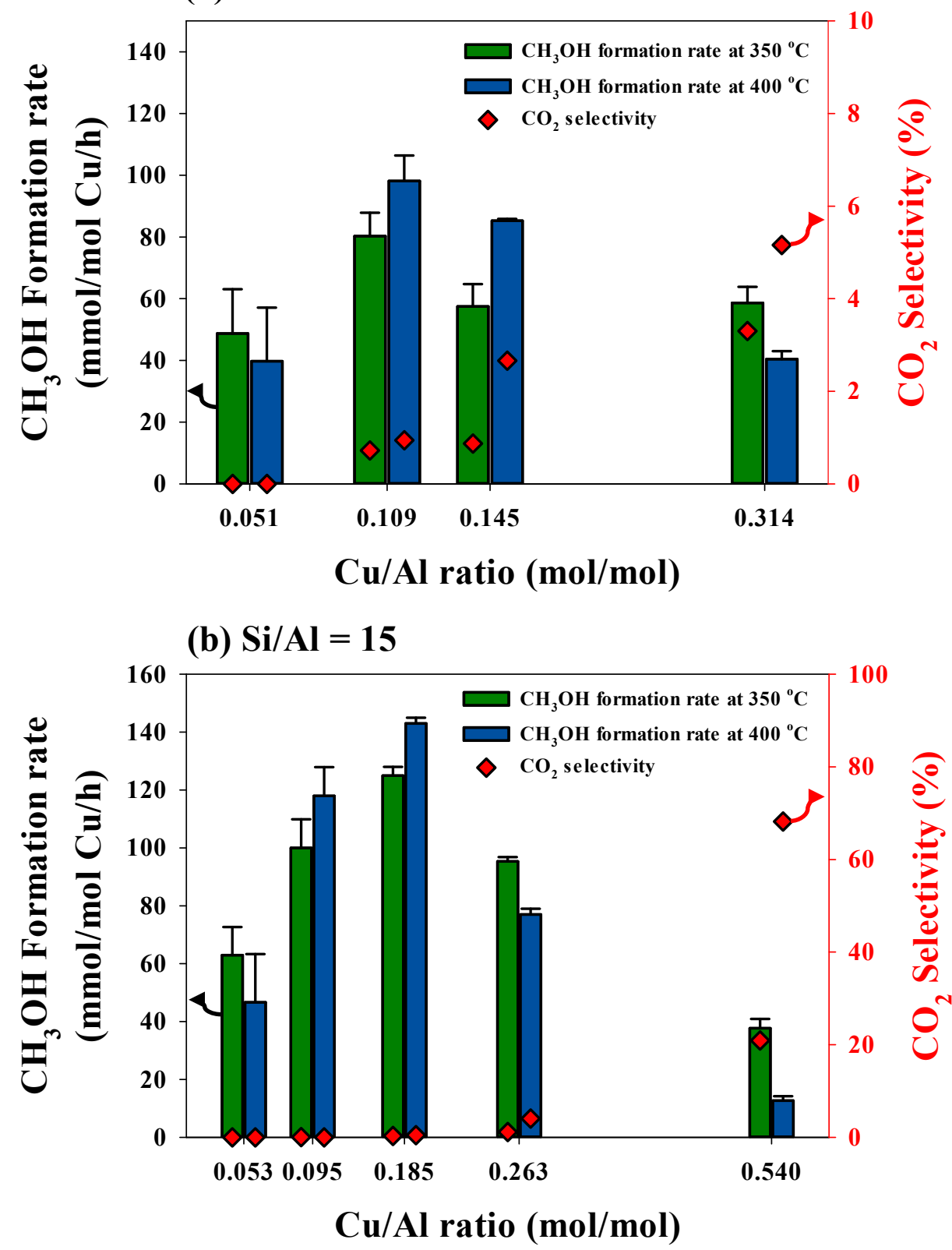

Figure S3. Effect of $\mathrm{Cu} / \mathrm{Al}$ ratio on continuous methane conversion over $\mathrm{Cu}-\mathrm{H}-\mathrm{MOR}$ (7.5) (a) and $\mathrm{Cu}-\mathrm{H}-\mathrm{MOR}(15)$ (b). Bar chart shows average methanol formation rate at $350{ }^{\circ} \mathrm{C}$ (green) and $400{ }^{\circ} \mathrm{C}$ (blue). Feed was composed of 33 mol\% methane, $85 \mathrm{ppm} \mathrm{O}_{2}$ and $67 \mathrm{~mol} \%$ steam. Error bars represent standard deviation. 

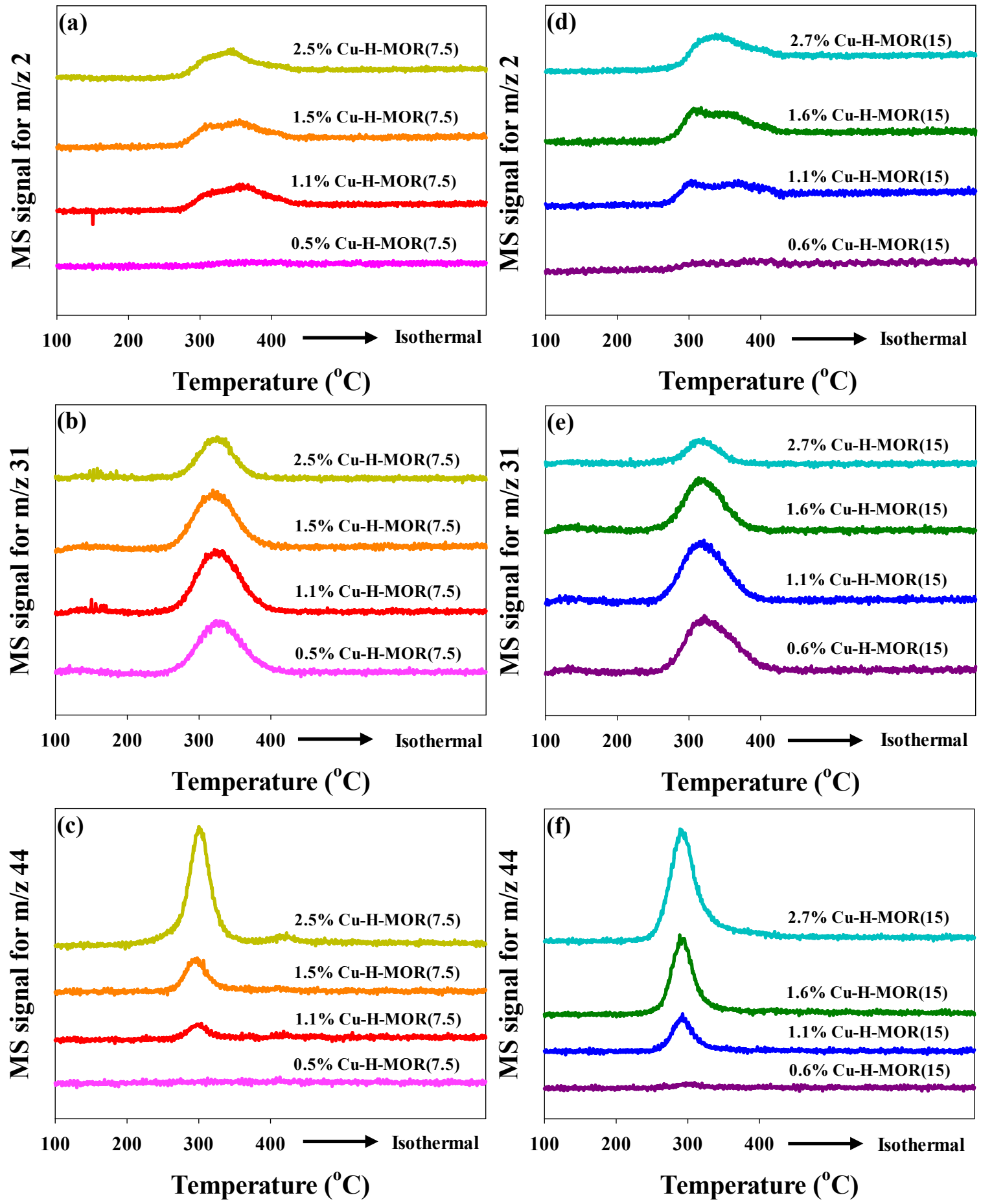

Figure S4. Temperature-programmed surface reactions with mass spectroscopy (TPSRs-MS) patterns of Cu-HMOR with different $\mathrm{Cu}$ contents and $\mathrm{Si} / \mathrm{Al}$ ratios. (Cu-H-MOR(7.5) (a, b, c) and $\mathrm{Cu}-\mathrm{H}-\mathrm{MOR}(15)(\mathrm{d}, \mathrm{e}, \mathrm{f})$. (a), (d) $\mathrm{m} / \mathrm{z}=2$ (hydrogen) (b), (e) $\mathrm{m} / \mathrm{z}=31$ (methanol) (c), (f) $\mathrm{m} / \mathrm{z}=44$ (carbon dioxide) 


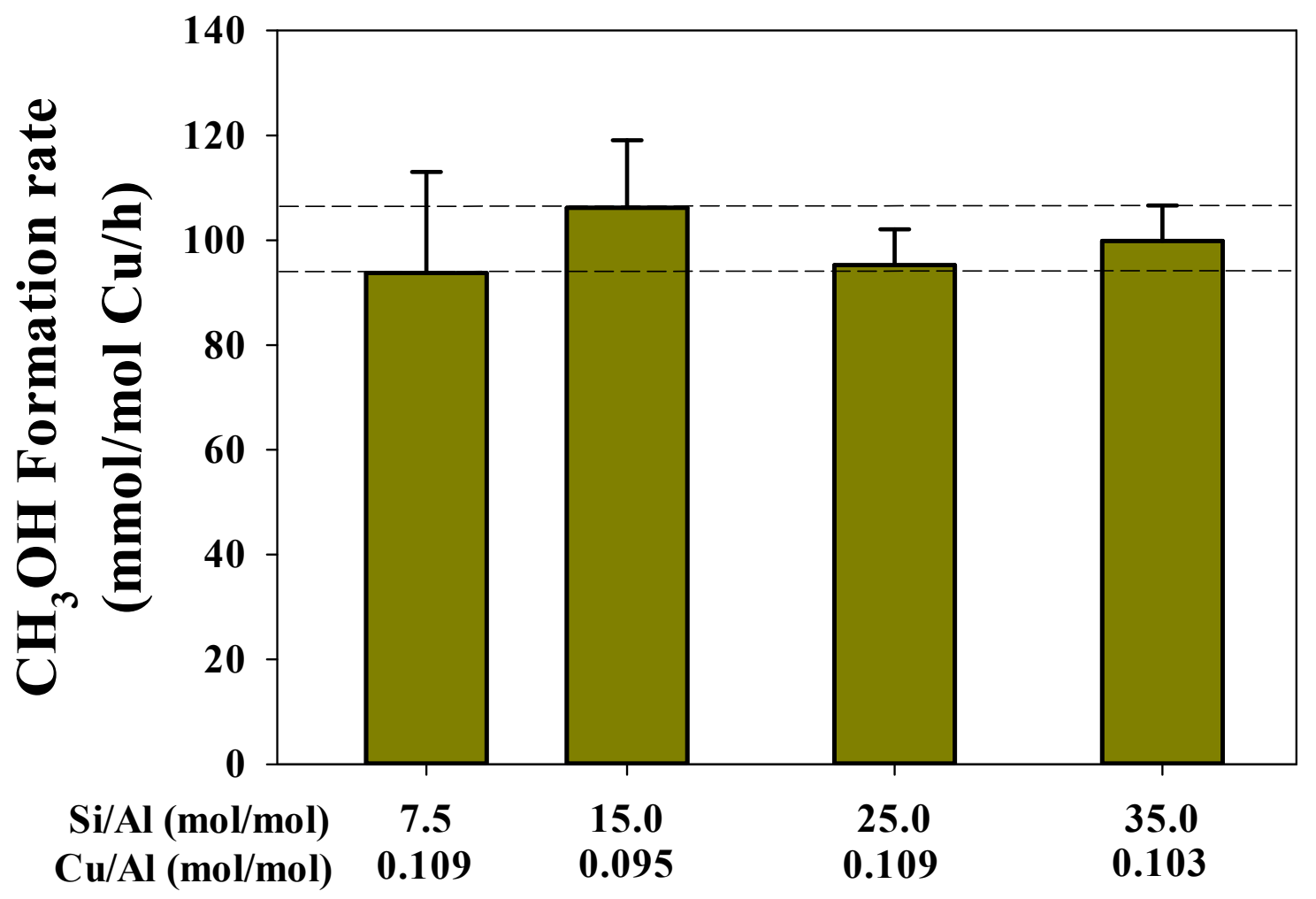

Figure S5. The effect of $\mathrm{Si} / \mathrm{Al}$ ratio in $\mathrm{H}-\mathrm{MOR}$ on the continuous methanol synthesis at $350{ }^{\circ} \mathrm{C}$ from methane and steam over $\mathrm{Cu}-\mathrm{MOR}$ with similar $\mathrm{Cu} / \mathrm{Al}$ ratios. The bar chart means average of methanol formation rate and error bar is a standard deviation. The feed is composed of $33 \mathrm{~mol} \%$ methane, $85 \mathrm{ppm} \mathrm{O}_{2}$ and $67 \mathrm{~mol} \%$ steam. 


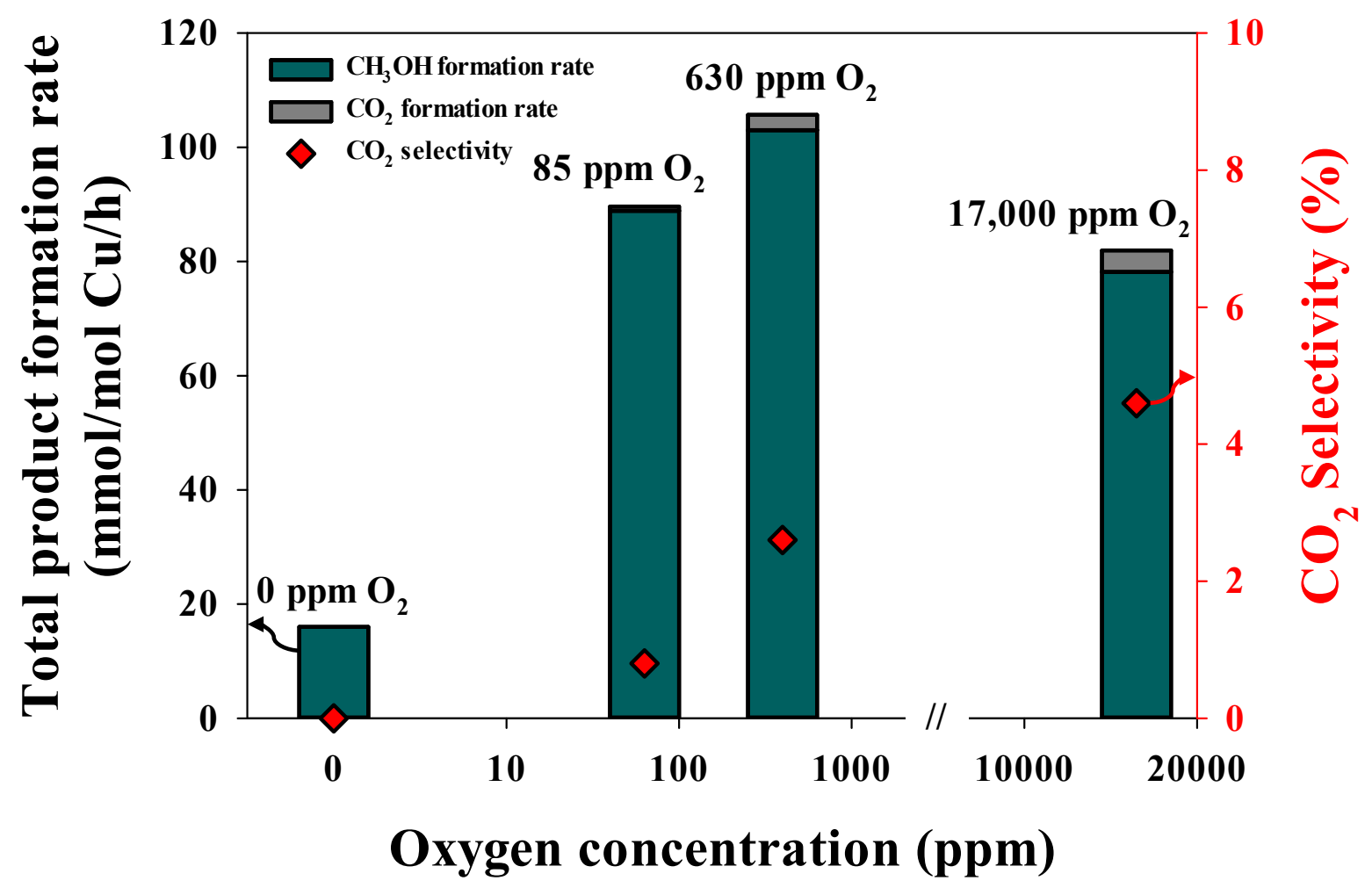

Figure S6. Effect of $\mathrm{O}_{2}$ concentration in the feed stream on continuous methane conversion over $1.1 \% \mathrm{Cu}-\mathrm{H}-$ MOR (7.5) at $350{ }^{\circ} \mathrm{C}$. 


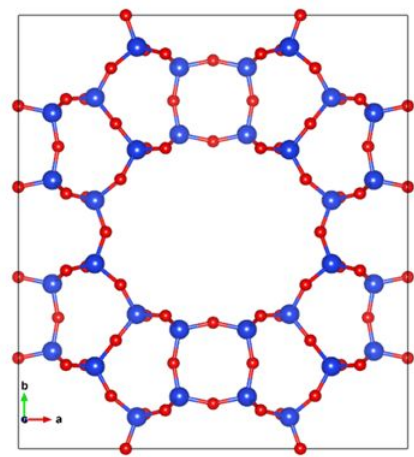

MOR

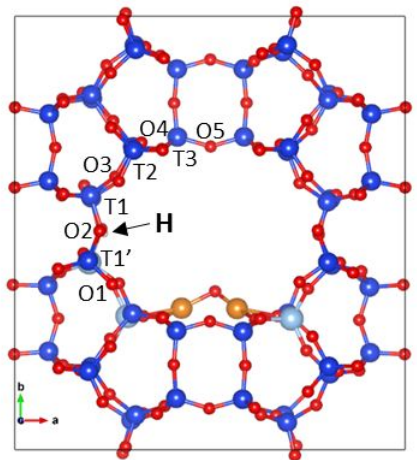

H-Cu-MOR

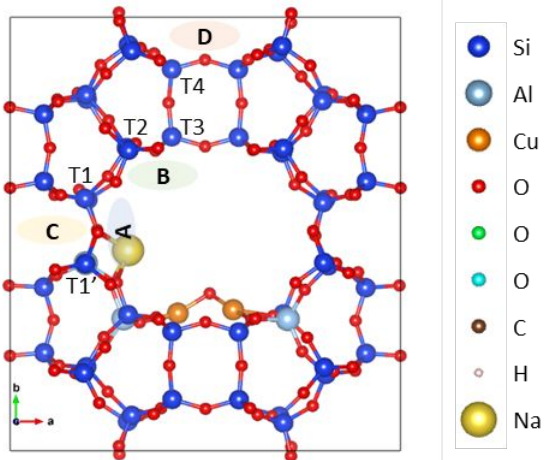

$\mathrm{Na}-\mathrm{Cu}-\mathrm{MOR}$

Figure S7. Substituting $\mathrm{Si}$ with $\mathrm{Al}$ in the initial model $(\mathrm{Al} / \mathrm{Si}=0)$ allowed the doping of $\mathrm{H}, \mathrm{Na}$ and $\mathrm{Cu}$ due to the charge compensation effect ${ }^{[1]}$. To investigate $\mathrm{Al}$ substitution and lattice cation positions based on Mortier site ${ }^{[2]}$, the above models were selected. Although $\mathrm{Na}$ was the most stable at the $\mathrm{C}$ site, the second most stable $\mathrm{A}$ site was chosen to investigate the effects of $\mathrm{Na}$ located near the active species. 


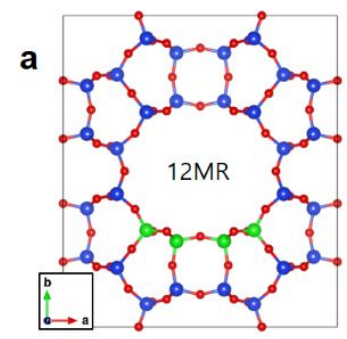

b

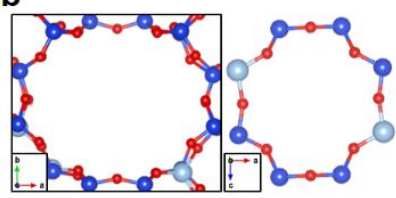

$E_{\mathrm{ret}}=0.00 \mathrm{eV}$

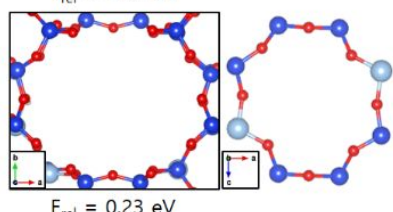

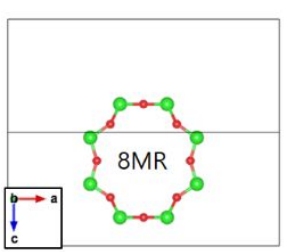

C
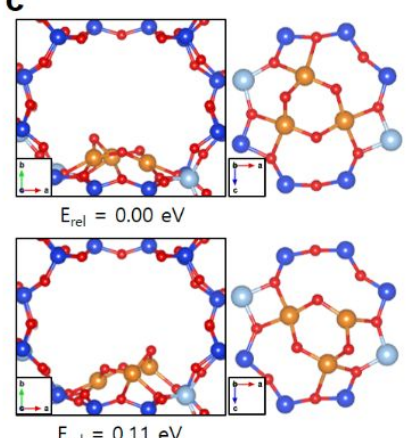
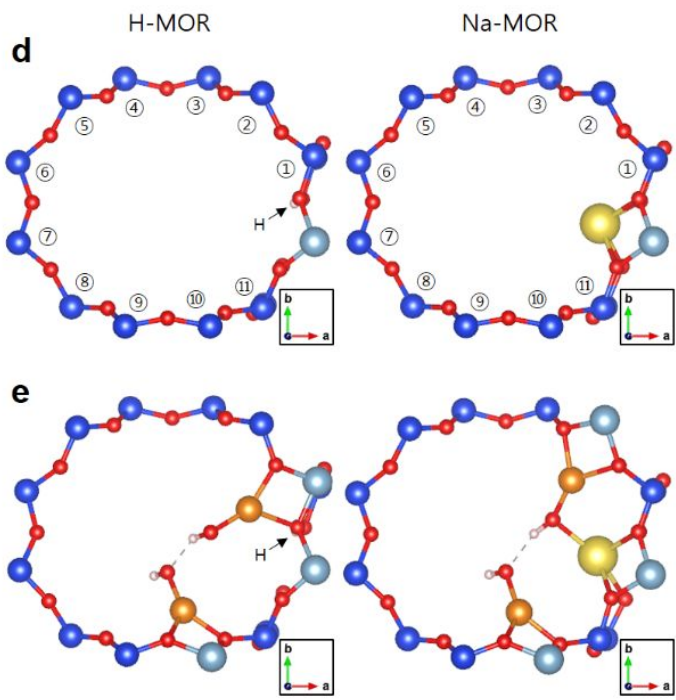

Figure S8. (a) Mordenite has 8-membered-ring (8MR) and 12-membered-ring (12MR). Dimeric and trimeric active species were generated at $8 \mathrm{MR}$, but monomeric active species could not be modeled at $8 \mathrm{MR}$. Because two or more copper atoms must be present for the two-electron reaction (methane-methanol conversion and copper re-oxidation), but placing two monomeric species in $8 \mathrm{MR}$ forms a bridging species. Once bridging species are formed, proton transfer does not occur efficiently. (b) Dimeric and trimeric copper species were based on two Al substitutions, and the two cases were compared energetically due to the periodicity of a unit cell. (c) From the structure determined in (b), the dimeric species could have only one structure, but the trimeric species could have two structures, of which energy-favorable one was chosen. (d) When two of the $11 \mathrm{Si}$ atoms remaining in the 12MR of H-MOR and Na-MOR were replaced with $\mathrm{Al}$ atoms, (1)-(10) and (2)-(10) combinations were the most stable. (e) The two monomeric species model interacted with each other and formed a stable structure. 

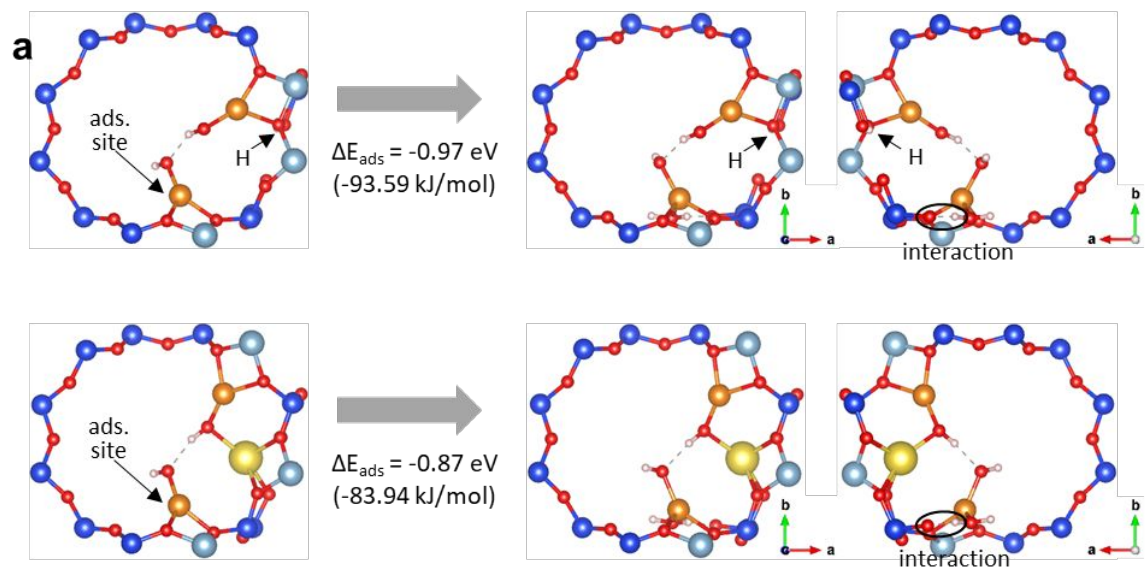

b

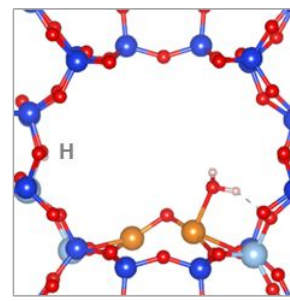

$\Delta \mathrm{E}_{\mathrm{ads}}=-0.76 \mathrm{eV}$

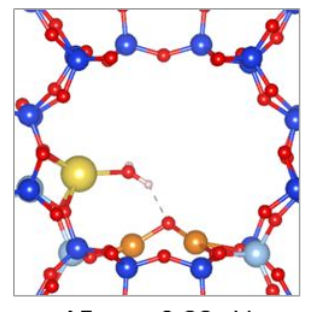

$\Delta \mathrm{E}_{\mathrm{ads}}=-0.88 \mathrm{eV}$
C
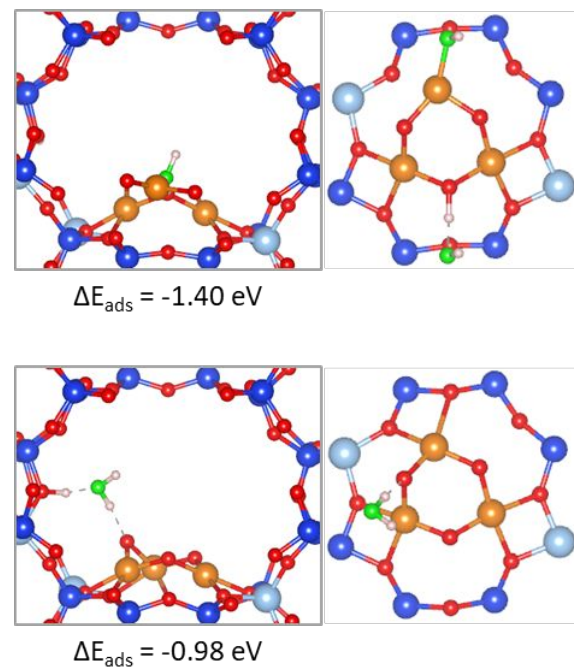

Figure S9. Adsorption sites and energetics of water according to lattice cations and active species were investigated.

(a) In case of monomeric species, the water molecule was adsorbed to the back of the copper (10) and the $\mathrm{H}$ of water became stable by interacting with the lattice oxygen behind 12MR. The adsorption energy of water was $-0.97 \mathrm{eV}$ in $\mathrm{Cu}-\mathrm{H}-\mathrm{MOR}$ and $-0.87 \mathrm{eV} \mathrm{Cu}-\mathrm{Na}-\mathrm{MOR}$. (b) In case of dimeric species, the $\mathrm{H}_{2} \mathrm{O}$ adsorbed to copper in $\mathrm{Cu}-\mathrm{H}-\mathrm{MOR}$ and to $\mathrm{Na}$ atom in $\mathrm{Cu}-\mathrm{Na}-\mathrm{MOR}$. The adsorption energies were $-0.76 \mathrm{eV}$ and $-0.88 \mathrm{eV}$, respectively, indicating that water adsorbed more strongly on $\mathrm{Cu}-\mathrm{Na}-\mathrm{MOR}$. (c) $\mathrm{H}_{2} \mathrm{O}$ was the most energetically stable when decomposed by the oxo structure of neighboring cells. However, the below structure with molecular $\mathrm{H}_{2} \mathrm{O}$ adsorption was selected although relatively unstable, because the $\mathrm{H}_{2} \mathrm{O}$ dissociation was occurred due to neighboring trimeric copper species. In addition, it was our interest to observe the reactions in the system where non-dissociative $\mathrm{H}_{2} \mathrm{O}$ molecules are present. Water was strongly adsorbed on $\mathrm{Cu}-\mathrm{H}-\mathrm{MOR}$ in order of trimeric, monomeric and dimeric species. 


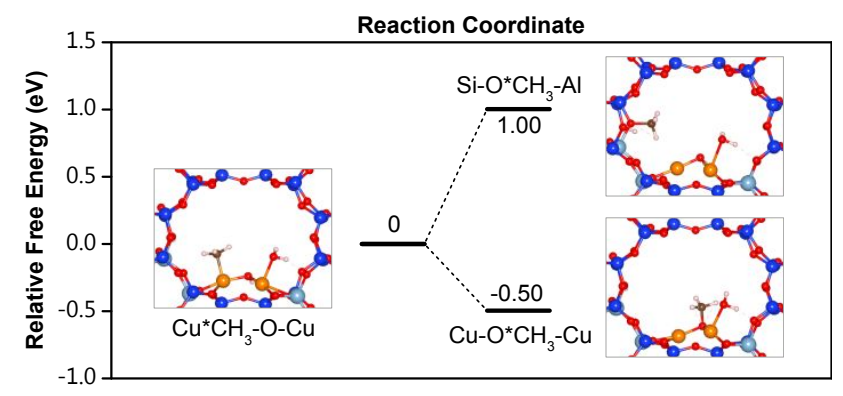

Figure S10. Relative energies and corresponding structures of the methoxy formation pathway. The pathway for $\mathrm{Cu}-\mathrm{OCH}_{3}-\mathrm{Cu}$ formation was more stable than the pathway for SMG formation near the Brønsted acid sites $\left(\mathrm{Si}_{-}-\mathrm{OCH}_{3}-\mathrm{Al}\right)$ by $1.50 \mathrm{eV}$, and we treated the more stable one as the methoxy intermediate in this work. For reaction steps other than the formation of methoxy species, we constructed various pathways and models and selected the most stable one. 


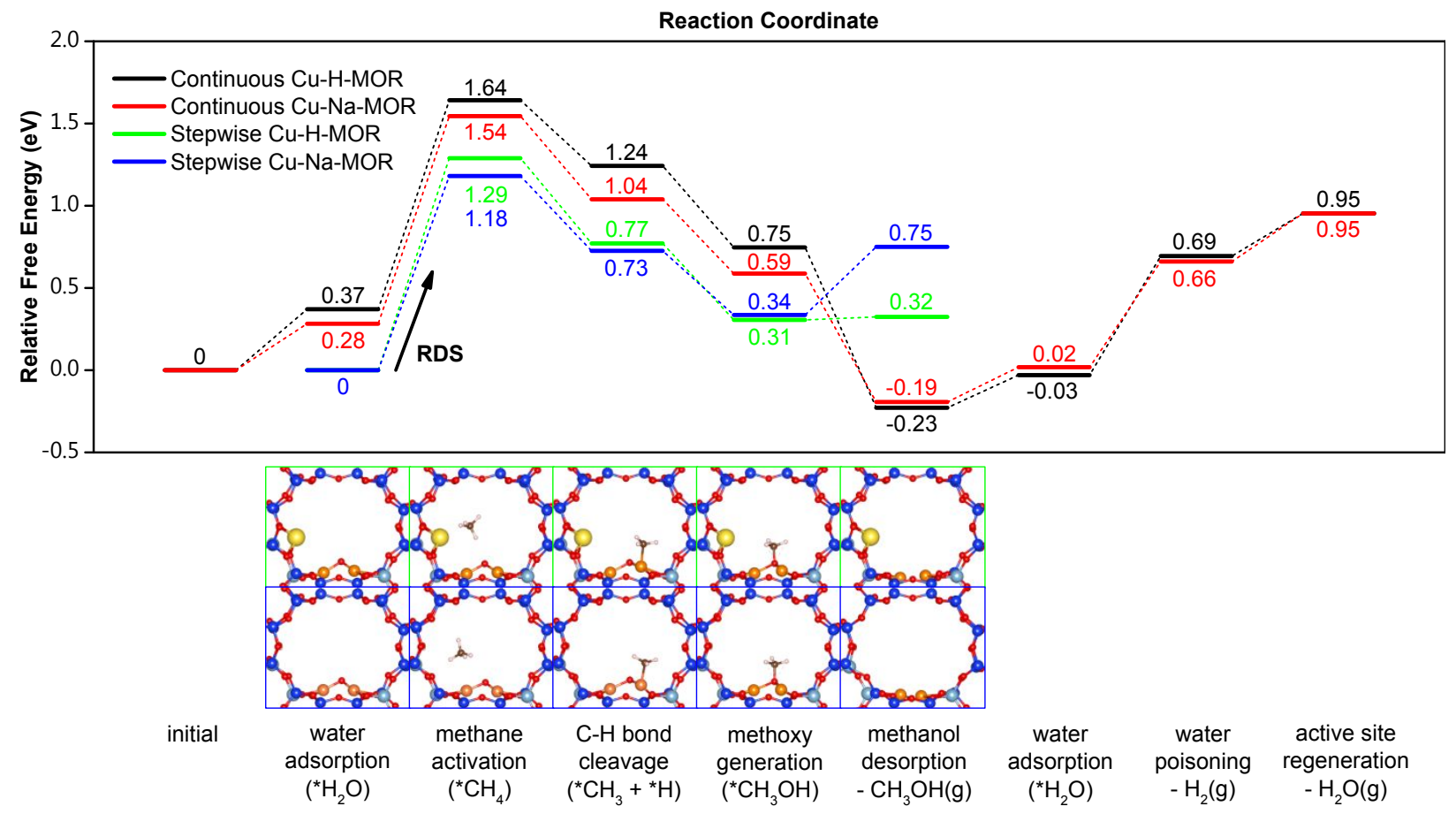

Figure S11. Energy profiles and corresponding structures for continuous (with $\mathrm{H}_{2} \mathrm{O}$ ) and stepwise (without $\mathrm{H}_{2} \mathrm{O}$ ) methanol conversion over dimeric copper species in $\mathrm{Cu}-\mathrm{H}-\mathrm{MOR}$ and $\mathrm{Cu}-\mathrm{Na}-\mathrm{MOR}$. In $\mathrm{Cu}-\mathrm{H}-\mathrm{MOR}$, the methane activation step became slightly more difficult $(0.02 \mathrm{eV})$ in the absence of water, but was almost the same. In Cu-Na-MOR, the methane activation step became easier by $0.08 \mathrm{eV}$ in the absence of water. $\mathrm{Cu}-\mathrm{Na}-\mathrm{MOR}$ adsorbed methane most strongly near $\mathrm{Na}^{+}$during the stepwise methane-to-methanol conversion. However, when there was a $\mathrm{H}_{2} \mathrm{O}$ molecule in the system, $\mathrm{H}_{2} \mathrm{O}$ was adsorbed to $\mathrm{Na}^{+}$instead of methane. The blocked methane was adsorbed to copper site. $\mathrm{Cu}-\mathrm{H}-\mathrm{MOR}$ adsorbed methane with similar binding strength to the copper active point regardless of the presence of steam. The methanol production step was largely spontaneous in the presence of $\mathrm{H}_{2} \mathrm{O}$ molecule. However, without water, the methanol production step became more difficult by 0.99 and $1.19 \mathrm{eV}$ in $\mathrm{Cu}-\mathrm{H}-\mathrm{MOR}$ and $\mathrm{Cu}-\mathrm{Na}-\mathrm{MOR}$, respectively. The system after methanol desorption could be stabilized by filling the empty active site with water molecules. In addition, these filled copper active sites could be regenerated by receiving oxygen from water molecules. 

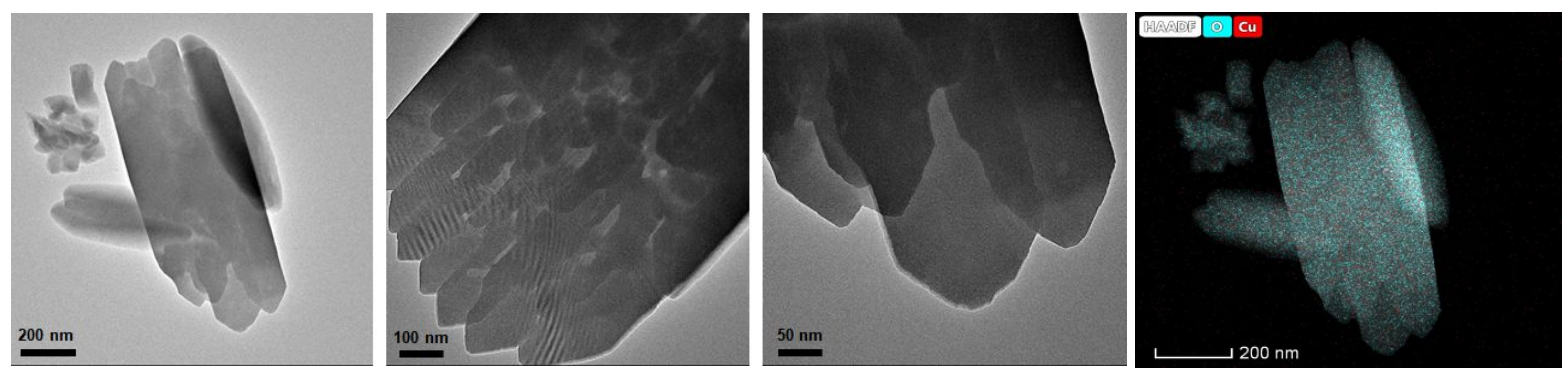

Figure S12. Transmission electron microscopy (TEM) images and mapping with $\mathrm{Cu}$ for $0.3 \mathrm{wt} \% \mathrm{Cu}-$ MOR(15). These data support the absence of $\mathrm{Cu}$ nanoparticles which might be able to cause steam reforming and subsequent water-gas shift reactions. Tecnai $\mathrm{G}^{2} 20$ S-TWIN TEM with high angle annular dark field detector (HAADF) and an energy dispersive x-ray spectrometer (EDS) was used.

\section{Reference}

(1) Chibani, S.; Chebbi, M.; Lebègue, S.; Bučko, T. A DFT investigation of the adsorption on iodine compounds and water in H-, Na-, Ag-, and Cu-mordenite. J. Phys. Chem. 2016, 144, 244705.

(2) Pluth, J. J.; Smith, J. V.; Mortier, W. J. POSITIONS OF CATIONS AND MOLECULES IN ZEOLITES WITH THE CHABAZITE FRAMEWORK. IV HYDRATED AND DEHYDRATED Cu2+-EXCHAGED CHABAZITE. Mat. Res. Bull 1977, 12, 1001-1007.

\section{Author Contributions}

Y.R. Jeong prepared all the catalysts and obtained the activity data. H. Jung carried out the DFT calculation. J. Kang characterized the catalysts. Prof. J.W. Han designed the DFT calculation. Prof. E.D. Park designed this investigation and wrote the original draft with Prof. J.W. Han. 